\title{
Collecting sexual orientation and gender identity information in the emergency department : the divide between patient and provider perspectives
}

\author{
Lisa M Kodadek, ${ }^{1}$ Susan Peterson, ${ }^{2}$ Ryan Y Shields, ${ }^{3}$ Danielle German, ${ }^{4}$ Anju Ranjit, ${ }^{5}$ \\ Claire Snyder, ${ }^{6,7}$ Eric Schneider, ${ }^{8}$ Brandyn D Lau, ${ }^{9,10,11,12}$ Adil H Haider $^{5}$
}

- Additional material is published online only. To view, please visit the journal online (http://dx.doi.org/10.1136/ emermed-2018-207669).

For numbered affiliations see end of article.

\section{Correspondence to}

Dr Lisa M Kodadek, Department of Surgery, Vanderbilt University Medical Center, Nashville TN 37212, USA;

lisa.kodadek@vumc.org

This work was presented in abstract form at the American College of Emergency Physicians (ACEP) meeting in Boston, Massachusetts in October 2015

Received 3 April 2018 Revised 29 October 2018 Accepted 29 November 2018 Published Online First 10 January 2019

\section{Linked}

- http://dx.doi.org/10.1136/ emermed-2018-208240

\section{Check for updates}

(C) Author(s) (or their employer(s)) 2019. No commercial re-use. See rights and permissions. Published by BMJ.

To cite: Kodadek LM,

Peterson S, Shields RY,

et al. Emerg Med J

2019:36:136-141.

\begin{abstract}
Background In the USA, The Joint Commission and Institute of Medicine have called for collection of patient sexual orientation (SO) and gender identity (GI) information in healthcare. In a recent study, we reported that ED clinicians believe patients will refuse to provide this information; however, very few patients say they would refuse to provide SO/GI. As part of this study, we interviewed patients and providers regarding the importance of collecting this information. While these interviews were briefly summarised in our prior report, the qualitative data warranted a more thorough analysis and exposition to explore provider and patient views as well as risks and benefits of collecting SO/GI.

Methods A purposive sample of 79 participants was recruited for semi-structured interviews between August 2014 and January 2015. Participants included community members who had a previous ED encounter and ED providers from 3 community and 2 academic centres in a major US metropolitan area. Interviews were conducted one-on-one in person, audio-recorded and transcribed verbatim. Data were analysed using the constant comparative method.

Results Fifty-three patients and 26 ED providers participated. Patients perceived collection of SO/GI to

\section{Key messages}

What is already known on this subject

- Initiatives across the world have called for collection of patient sexual orientation and gender identity (SO/GI) information in healthcare to address sexual and gender minority (SGM) health disparities.

- We previously reported that ED clinicians believe patients will refuse to provide this information; however, we found that very few patients reported they would refuse to do so.

What this study adds

- This manuscript describes the in-depth findings of our qualitative interviews to contrast the differing perspectives of patients and providers in the importance of gathering SO/GI information.

- ED clinicians feel that SO/GI is not usually relevant but patients feel routine SO/GI collection allows for recognition of individual identity and improved therapeutic relationships in the ED.
\end{abstract} be important in most clinical circumstances because $\mathrm{SO} /$ $\mathrm{Gl}$ is relevant to their identity and allows providers to treat the whole person. However, many providers felt SO/GI was not relevant in most clinical circumstances because similar care is provided to all patients regardless of SO/GI. Patients and providers agreed there are risks associated with collecting SO/GI in the ED.

Conclusions ED clinicians do not perceive routine collection of $\mathrm{SO} / \mathrm{Gl}$ to be medically relevant in most circumstances. However, patients feel routine SO/GI collection allows for recognition of individual identity and improved therapeutic relationships in the ED. These discordant perspectives may be hindering patient-centred care, especially for sexual and gender minority patients.

\section{INTRODUCTION}

Research to understand health outcomes and disparities among sexual and gender minorities (SGM), including lesbian, gay, bisexual and transgender individuals, is limited. Previous studies that have collected sexual orientation and gender identity (SO/GI) among small cohorts of patients in the ambulatory setting have identified disparities that exist for SGM. ${ }^{1-6}$ Data from EDs and inpatient settings are lacking. ${ }^{7}$ Recently, several US institutions, including the Department of Health and Human Services, the Centers for Medicare and Medicaid Services, the National Academy of Medicine and The Joint Commission have recognised the importance of identifying disparities among SGM patients. ${ }^{8-11}$

$\mathrm{SO} / \mathrm{GI}$ data are not routinely collected in healthcare settings worldwide making it difficult to conduct research into disparities based on gender identity and sexual orientation. While recommendations to collect SO/GI information exist, few institutions collect these data and no evidencebased guidelines exist. With high throughput and a diverse patient base, the ED is poised to provide leadership and inform policy surrounding SO/GI collection in various healthcare settings nationwide and throughout the world. However, to ensure successful implementation of SO/GI collection, it is important to understand the perspectives of key stakeholders, which include patients and providers. In a previous report, ${ }^{12}$ we demonstrated that patients and providers have divergent views on the importance of collecting this information. While we have recently published brief initial qualitative findings from the initial phase used to inform the 
overall project strategy, ${ }^{12}$ the purpose of this current report is to provide in-depth exploration of patient and provider perspectives concerning the importance of collection of patient SO/GI in the ED.

\section{METHODS}

\section{Study design, setting and selection of participants}

This study is part of a multiphase mixed methods research programme known as Emergency Department Query for Patient-Centred Approaches to Sexual Orientation and Gender Identity, ${ }^{12}$ which seeks to identify and test the optimal patient-centred way to collect SO/GI data. This study recruited ED providers and adult patients from the greater Baltimore and Washington DC metropolitan area. ED providers were defined as nurses, physician assistants or physicians in current emergency medicine practice. Patients were recruited using flyers, social media and community outreach. Providers were recruited by email from two academic and three community hospital EDs. Purposive sampling ensured a richly diverse group of participants. Specifically, patients were sampled with a goal to enrol lesbian, gay, bisexual and straight patients, as well as cisgender and transgender patients. Although 10 patients per group was set as an a priori goal, final enrolment was determined by ability to achieve thematic saturation of the data. There were no specific enrolment demographic goals for providers. Providers identifying as SGM were neither specifically recruited nor excluded from participation. All participants gave written informed consent and received a US\$35 gift card as remuneration for their time.

\section{Study protocol}

A semi-structured interview guide was developed for patients and providers (online supplementary appendix). Questions probed various domains related to collection and use of SO/GI in the ED. The interview guide was developed with a 26 -member national Stakeholder Advisory Board (SAB) comprising providers, patients, community advocates and SGM health experts. The interview guide was piloted prior to use and revised in an iterative fashion to ensure all relevant topics were explored. Interviews were conducted between August 2014 and January 2015 by two experienced qualitative researchers. Interviews proceeded during the period of data analysis and additional participants were sought until theoretical saturation was achieved, or no new themes emerged. All interviews were conducted one-on-one in person in a private location with audio recording. The average interview duration for patients was 1 hour and for providers was $30 \mathrm{~min}$. Summative notes were taken during and after the interviews. An independent Health Insurance Portability and Accountability Act (HIPAA) compliant professional transcriptionist reviewed and transcribed audio recordings verbatim.

\section{Data analysis}

Data analysis was conducted using the constant comparative method. ${ }^{13}$ Two investigators read transcripts independently and identified general topics through open coding. By comparing open coding notes, the investigators developed a codebook of the preliminary taxonomy of themes. The codebook was applied to the transcripts allowing for some codes to be collapsed and others expanded to ensure accurate representation of the data. The final codebook was applied to every transcript and data were organised using ATLAS.ti (V.1.0.0 (66), Berlin, Germany) software. The two investigators met regularly to review data and to identify recurrent patterns and emerging themes. A third investigator with expertise in qualitative methodology and SGM health provided oversight. Discrepancies were arbitrated with the investigative team and the SAB. All investigators engaged in reflexive discussion throughout the study to limit bias. ${ }^{14}$ Recurrent and unifying themes were presented to the SAB to check accuracy and consistency.

\section{RESULTS}

\section{Characteristics of study subjects}

Fifty-three patient participants and 26 provider participants were interviewed, details of whom have been reported previously. ${ }^{12}$ Demographics are provided in table 1.

\section{Main results}

In-depth qualitative analysis revealed nuances of two major areas of thematic discordance between patients and providers concerning: (1) the perceived medical relevance of SO/GI in the ED and (2) the role of recognising the identity of individual patients (table 2).

Although some providers recognised a role for SO/GI collection to enhance individual patient care, most providers felt $\mathrm{SO} /$ GI was not relevant in the majority of circumstances because they felt similar care is provided to all patients regardless of $\mathrm{SO} / \mathrm{GI}$. While some patients voiced risks related to privacy or consequences of disclosure, most patients perceived collection of SO/GI to be important in all circumstances, because SO/GI is relevant to their identity as a person and disclosure enhances patient-centred care. Table 3 provides a summary of findings using representative quotations.

\section{Medical relevance of SO/GI information}

Most patients felt that SO/GI was always relevant to the healthcare encounter regardless of the clinical concern. Both patients and providers recognised the importance of SO/GI when the patient presented with a reproductive system issue. However, providers did not recognise SO/GI as medically relevant in the majority of cases. For example, one ED physician commented: "Your sexual orientation means nothing to me if you have a headache...it's your business. I don't really care so it's never going to impact how I care for you". Many providers did not feel patients' SO/GI was necessary information unless perceived to be clinically relevant. In contrast, patients felt their healthcare provider always needed to know SO/GI to provide the best care. A bisexual cisgender man commented, "I would feel like if they are trying to treat me that it's really their business to know". While SGM patients understood the medical relevance of SO/ GI in the ED, many non-SGM patients also understood the need to collect this information. A cisgender heterosexual woman commented, 'Knowing that information (SO/GI) might be critical to the way (patients) are treated'. Other non-SGM patients suggested that asking all ED patients about SO/GI is necessary because providers are not able to assume this information. Furthermore, patients recognised collection of SO/GI might be helpful to understand social aspects of a patient's presentation.

Transgender patients had specific clinical reasons why their provider would need to know their GI and sex assigned at birth. Many transgender patients receive medical and/or surgical treatments and wanted to ensure their provider was aware. For example, a queer-identified transgender man commented, "and that comes down to even like testosterone...I need to make sure medications I'm prescribed don't interact with that". While some transgender patients preferred not to disclose sex assigned at birth, the majority felt it was important and nearly always 


\begin{tabular}{|c|c|}
\hline Patient characteristics $(n=53)$ & Percentage $(\mathrm{N})$ \\
\hline \multicolumn{2}{|l|}{ Gender identity } \\
\hline Cisgender men & $30(16)$ \\
\hline Cisgender women & $40(21)$ \\
\hline Transgender men & $5.5(3)$ \\
\hline Transgender women & $17(9)$ \\
\hline Genderqueer/bigender & $7.5(4)$ \\
\hline \multicolumn{2}{|l|}{ Sexual orientation } \\
\hline Lesbian & $17(9)$ \\
\hline Gay & $23(12)$ \\
\hline Bisexual & $23(12)$ \\
\hline Queer & $3.7(2)$ \\
\hline Straight/heterosexual & $26(14)$ \\
\hline Other* & $7.5(4)$ \\
\hline \multicolumn{2}{|l|}{ Race } \\
\hline White/Caucasian & $43(23)$ \\
\hline Black/African-American & $45(24)$ \\
\hline Asian & $9.4(5)$ \\
\hline Not listed & $1.8(1)$ \\
\hline \multicolumn{2}{|l|}{ Ethnicity } \\
\hline Hispanic/Latino(a) & $8(4)$ \\
\hline Not Hispanic/Latino(a) & $92(49)$ \\
\hline \multicolumn{2}{|c|}{ Highest level of education completed } \\
\hline High school & $13(7)$ \\
\hline Associates/trade school & $7.5(4)$ \\
\hline Some college & 11 (6) \\
\hline Bachelors & $32(17)$ \\
\hline Advanced degree & $21(11)$ \\
\hline Not listed & $15(8)$ \\
\hline \multicolumn{2}{|l|}{ Age (years) } \\
\hline$<30$ & $28(15)$ \\
\hline $30-39$ & $15(8)$ \\
\hline $40-49$ & $19(10)$ \\
\hline $50-59$ & $26(14)$ \\
\hline $60+$ & $11(6)$ \\
\hline
\end{tabular}

\begin{tabular}{ll}
\hline Provider characteristics ( $\mathbf{n} \mathbf{2 6}$ ) & Percentage (N) \\
\hline Gender identity & \\
$\quad$ Cisgender men & $35(9)$ \\
$\quad$ Cisgender women & $65(17)$ \\
\hline $\begin{array}{l}\text { Sexual orientation } \\
\text { Straight/heterosexual }\end{array}$ & $100(26)$ \\
Role in ED & \\
$\quad$ Physician & $35(9)$ \\
\hline Physician assistant & $19(5)$ \\
\hline Nurse & $46(12)$ \\
\hline Race & \\
\hline White/Caucasian & $69(18)$ \\
\hline Black/African-American & $12(3)$ \\
\hline Asian & $15(4)$ \\
\hline Pacific Islander & $4(1)$ \\
\hline Ethnicity & \\
\hline Hispanic/Latino(a) & $4(1)$ \\
\hline Not Hispanic/Latino(a) & $96(25)$ \\
\hline Age (years) & $23(6)$ \\
\hline$<30$ & $38(10)$ \\
\hline $30-39$ & $19(5)$ \\
\hline $40-49$ & \\
\hline & \\
\hline
\end{tabular}

Table 1 Continued

\begin{tabular}{lc}
\hline Provider characteristics $(\mathbf{n}=\mathbf{2 6})$ & Percentage $(\mathrm{N})$ \\
\hline $50-59$ & $8(2)$ \\
$60+$ & $12(3)$ \\
\hline
\end{tabular}

*Other category includes participants that reported being pansexual, asexual and not sure.

necessary to ensure the best care. Furthermore, since many transgender patients have legal names or documentation discordant with their gender identity, it was necessary to disclose GI to resolve any confusion and ensure proper names and pronouns were used during the visit.

\section{Recognition of individual patient identity}

Many providers felt SO/GI was not relevant to the clinical encounter because they valued treating all patients the same, regardless of SO/GI. Patients, however, saw value in being recognised as individuals. For example, an ED physician explained his approach to SGM patients, "If I suspect that's the case, I make it a point to treat this person the way I would anyone else and you know the significant other the way I would anyone else". Many providers valued treating all patients similarly, and felt that by taking an objective approach to their patients, they would protect against discrimination. One nurse commented, "If you would take the sex out of it, you have a human body that has a medical problem. It makes no difference what your sexual orientation is to treat that problem". While seeing the patient as a human body may be sufficient to address medical problems, patients suggested that seeing the patient as a person may improve the patient-centredness of care. To effectively treat a patient for an emergent illness, it was important for the provider to recognise the patient as a whole person, engage the patient's caregivers and avoid inappropriate assumptions about SO/GI. When providers assumed SO/GI incorrectly, for example, through inaccurate pronoun references to partner gender, SGM patients sometimes felt pressured to disclose SO/ GI to correct the provider. Patients felt that collection of SO/GI from all patients in a standard fashion would promote inclusivity by removing the need for SGM patients to self-identify.

Most patients felt collection was needed for the benefit within the context of the medical encounter, and to demonstrate that the institution and providers expect and welcome SGM patients. A transgender patient explained that by routinely asking all patients about SO/GI, we remove a burden from individual SGM patients:

I think not having the burden on the patient to say I am different in this way is really important. To put it out there that yes, you're free to exist just like any other. We think of you when we think of our patients.

Table 2 Major discordance: patient vs provider perspectives

\begin{tabular}{ll} 
Patient perspectives & Provider perspectives \\
\hline $\begin{array}{l}\text { SO/GI disclosure facilitates patient-provider } \\
\text { rapport }\end{array}$ & $\begin{array}{l}\text { SO/GI disclosure not necessary } \\
\text { because SGM patients receive same } \\
\text { care as other patients }\end{array}$ \\
$\begin{array}{l}\text { SO/GI disclosure crucial for individual patient- } \\
\text { centred care }\end{array}$ & $\begin{array}{l}\text { Value treating all patients the same } \\
\text { regardless of SO/GI }\end{array}$ \\
$\begin{array}{ll}\text { SO/GI relevant regardless of specific medical } \\
\text { concern }\end{array}$ & $\begin{array}{l}\text { SO/GI not relevant unless patient } \\
\text { has reproductive system concerns }\end{array}$ \\
\hline GI, gender identity; SGM, sexual and gender minority; SO, sexual orientation.
\end{tabular}


Table 3 Patient vs provider perspectives concerning medical relevance and recognition of sexual orientation and gender identity data

Patient perspective: SO/GI relevant regardless of specific medical concern

\section{Representative quotations}

"I'm fine if they... ask me. You know, I wouldn't feel uncomfortable, and honestly, I would feel like if they are trying to treat me that it's really their business to know".

-Bisexual cisgender man

"Gender identity is relevant in terms of it takes medical steps for me to live as who I

$a m "$.

-Queer transgender man

"The relevancy... why is this so relevant? ...you've got to ask the questions (about SO/ GI) to everybody because you don't know them".

-Heterosexual cisgender man

Patient perspective: SO and GI disclosure facilitates patient-provider rapport and is crucial for patient-centred care

\section{Representative quotations}

"You cannot treat everybody the same, everybody is different, okay? That's like having more than one child. You can't treat each child the same, you have to treat them according to who they are".

-Bisexual cisgender woman

"You are supposed to have this psychosocially supportive relationship (between providers and patients). How can you do that if you don't know who this person is and where they are coming from?"

-Lesbian cisgender woman

Provider perspective: SO/GI not relevant unless there are reproductive system concerns

\section{Representative quotations}

"If I didn't have to, I wouldn't even broach on (SO and GI) unless they brought it up.. unless it affected some care that I was going to give them, that's their business".

-ED nurse

'The question is not always a relevant one for each individual patient'.

-ED physician

"(SO/GI) doesn't factor into anything that I need to do so I don't need to know it...it's not relevant to anything we do".

-ED nurse

"So if (SO/GI) is not clinically relevant, I wouldn't ask it".

-ED physician

Provider perspective: value treating all patients the same regardless of SO and GI

\section{Representative quotations}

"But as far as treating a patient whether they're gay, lesbian, bisexual for an STD, I'm going to treat them the same way as a straight person with an STD".

-ED nurse

"We're humans. Even though this is our sexual orientation, does that make you get less care or more care than me? Or different care? ...I don't see it making a difference in the care that somebody is going to get".

-ED nurse

$\mathrm{Gl}$ gender identity; SO, sexual orientation.

While providers valued treating patients similarly without consideration of SO/GI, many patients noted that 'treating all patients the same' equated to treating patients as if everyone is heterosexual and cisgender, which could lead to the continued marginalisation of SGM. A cisgender lesbian woman recognised the need to view patients as individual people:

I kind of feel like healthcare in the ideal setting...involves your mind, your body, your spirit and in order for it to do all those things your identity has to be celebrated or at the very least-acknowledged!

While patients appreciated the need for collection of SO/GI in healthcare settings for both individual identity recognition and societal benefit, many providers did not appreciate the importance of this practice. Some providers considered this discrimination and were concerned about offending patients or impairing the therapeutic relationship. However, patients described the need to acknowledge SO/GI as important to individualised care and a critical component of rapport.

\section{Risks and disadvantages of collecting SO/GI information}

Provider and patient participants verbalised risks and disadvantages with routine collection of SO/GI information including issues related to privacy, discrimination and workflow (table 4).
Patients were less willing to provide SO/GI in situations where privacy seemed compromised. For example, a patient commented on the physical treatment space: 'some emergency rooms have like the private rooms and others kind of like have the flex care where it is like the curtain system'. This issue became even more important when family members or friends accompanied the patient for care. In circumstances where the family was unaware of the patient's SO/GI, verbal disclosure became even more difficult. Patients had significant concern for discrimination, stigma and fear of worse care after disclosure. Some patient participants felt disclosure left them powerless and subject to the provider's bias. One patient verbalised his fear of discrimination: "Well I imagine that they will laugh at me, treat me different, treat me like I ain't (sic) nothing". Very few providers felt that there would be any serious consequences, although one nurse commented: "If I have to ask that, and if I'm asking the wrong person, that could be... a physical confrontation". However, the majority of participants did not think asking SO/GI represented a major risk. Many providers felt the practice of SO/GI collection would be time-consuming. Several providers felt that collecting these data in a sensitive manner would slow down ED workflow.

Table 4 Patient vs provider perspectives concerning risks of collecting SO and GI information

\section{Patient perspectives \\ Representative quotations}

"Yeah, and so it's always that question, what do you need it for? Why are you asking me? I don't trust this, you know, type of situation. The first thing that pops into your head is, are they judging me?"

-Bisexual cisgender woman

I: "Any risks with providing sexual orientation to a provider?" P: "Other than the fact that they may be homophobic or not want to treat you anymore, no. I mean, those are big risks, but I mean really if they're not homophobic and they take their oath and they say that they're going to treat you no matter what then there's really no risk to it". -Lesbian cisgender woman

\section{Provider perspectives}

Representative quotations

"I don't know if you know how our workflow is, but I don't think you're going to get the most honest results up front as you would if it was in a more private area". -ED nurse

"I could see that (disclosing SO/GI) being just a horrible event. And... if I were that patient I might feel discriminated against too...I think the risk is... if not asked properly and in a way that is respectful to the patient...the patient may also choose to not share other pertinent information".

-ED physician

GI, gender identity; I, interviewer; P, patient respondent; SO, sexual orientation. 


\section{Consequences of patient and provider discordant perspectives}

Patients recognised consequences surrounding these discordant patient and provider perspectives in healthcare settings. Many patients admitted that SGM are fearful about seeking emergency care secondary to challenging experiences with providers in the past. One transgender man recalled an experience in the ED, "It was clear that the nurses and providers weren't experienced in trans issues and made a lot of assumptions about who I was, using the wrong pronouns". In particular, patients explained that unlike in primary care settings, patients are unable to choose a provider in the ED who is knowledgeable about SGM health, which may serve as a barrier to emergency healthcare access. A bisexual cisgender woman commented, "What most (SGM) people do is they just don't go (to the ED)... a very large percentage will just not get care...they don't feel like they can trust the healthcare community". Many patients recognised that SGM often avoid seeking healthcare or delay care until acute illness worsens.

\section{Provider education opportunities}

Patients identified opportunities for education of ED providers. Providers also recognised the need to receive training on SGM health issues and many welcomed this opportunity. Patient participants agreed that cultural competency training and education about SO/GI minority health for providers would be an effective first step towards creating a more inclusive environment in the ED. Furthermore, by allowing ED providers to understand the patient perspective concerning the relevance of SO/GI in healthcare settings, higher quality care may be provided to all patients.

\section{DISCUSSION}

In this paper, we provide an in-depth exploration of differing perspectives among patient and providers concerning collection and use of SO/GI in the ED. While most patients recognise the relevance of SO/GI disclosure in healthcare settings, most providers do not consider SO/GI relevant in most instances of medical care. This provider perspective may prevent many patients, especially those who identify as SGM, from seeking and receiving individualised patient-centred care. ${ }^{15} 16$ Furthermore, patient-provider rapport and the therapeutic relationship may be compromised if SO/GI is ignored.

Providers report that collection of SO/GI is not important because all patients are treated the same regardless of SO/GI. Ironically, providers justify this approach by stating that it protects against discrimination. However, when providers indicate they treat all patients the same, this could equate to treating all patients as if they are heterosexual and cisgender. This approach fails to recognise patients as individual people with unique needs and perspectives.

Numerous US agencies, including the Institute of Medicine and The Joint Commission, recommend collection and documentation of SO/GI in healthcare settings. ${ }^{10} 11$ The prevailing argument for collecting this information has typically relied on a population-level benefit. Our results agree with others who have identified the benefits of routine collection of SO/GI in healthcare. ${ }^{17-20}$ However, our results also contribute new rationale that specifically addresses the patient perspective: collection of $\mathrm{SO} /$ GI allows patients to be recognised as individuals and improves patient-provider rapport. Furthermore, routine SO/GI collection demonstrates an inclusive environment and protects against harmful assumptions by the healthcare team. Finally, routine collection of SO/GI helps prevent invisibility among SGM within the healthcare system, health outcomes research and society. ${ }^{21}$

\section{Implications for practice}

These findings offer important insight about the discordant perspectives among patients and providers. A more nuanced understanding of the patient perspective can assist providers in their efforts to effect cultural change and provide more inclusive ED care for all patients in need. Lack of understanding may be hindering appropriate patient-centred care, especially for SGM. Others have also identified the importance of provider education and have encouraged incorporation of SGM health curriculum in healthcare provider education. ${ }^{22-24}$ Through education of healthcare providers, providers can recognise patients as individuals and ensure the highest quality patient-centred care for all patients in the ED. ${ }^{24}$

\section{LIMITATIONS}

Our findings represent the perspectives of our purposively sampled participants, but may not reflect the perspectives of patients and providers generally. Recruitment was focused within one US metropolitan area, and thus these findings may not be generalisable to all patients, especially those patients residing in other countries. Adults participated in this study and thus results are not generalisable to adolescent populations; further dedicated research may be necessary to understand adolescent perspectives concerning collection of SO/GI in the ED. Temporal differences in participant experiences may be present since the patients were not recruited during an ED visit; however, these temporal differences are not expected to uniformly influence the results in either a positive or negative direction. Selection bias may be present since all participants volunteered in response to recruitment materials outlining the topic of the study and all participants received a financial incentive for participation. Furthermore, those who participated are more likely to be comfortable disclosing SO/GI data in an ED setting. It should be noted that no provider participants identified as SGM. Divergence of patient and provider perspectives may be overestimated on account of this considerable limitation. As with other qualitative studies in which researchers collect data through interview techniques, the assumptions and interests of the researchers contribute to the results, but influence was minimised through explicit practice of reflexivity during the course of data acquisition and analysis.

\section{CONCLUSION}

Provider-perceived lack of medical relevance of SO/GI represents a barrier to individualised patient-centred care, especially for SGM. Many patients feel SO/GI is relevant to their healthcare and support a shift towards routine collection of this information. However, patients note that additional training of ED providers regarding SGM health is necessary to ensure the most effective therapeutic relationships. Routine collection of SO/GI may ultimately serve to provide better patient-centred care and provide necessary data to understand and improve the health of SGM patients.

\footnotetext{
Author affiliations

${ }^{1}$ Department of Surgery, Vanderbilt University Medical Center, Nashville, Tennessee, USA

${ }^{2}$ Department of Emergency Medicine, Johns Hopkins University School of Medicine, Baltimore, Maryland, USA

${ }^{3}$ Department of Obstetrics, Gynecology and Reproductive Sciences, Yale School of Medicine, New Haven, Connecticut, USA
} 
${ }^{4}$ Department of Health, Behavior and Society, Johns Hopkins Bloomberg School of Public Health, Baltimore, Maryland, USA

${ }^{5}$ Center for Surgery and Public Health, Harvard Medical School and Harvard T.H. Chan School of Public Health, Boston, Massachusetts, USA

${ }^{6}$ Department of Medicine, Johns Hopkins University School of Medicine, Baltimore, Maryland, USA

${ }^{7}$ Department of Health Policy and Management, Johns Hopkins Bloomberg School of Public Health, Baltimore, Maryland, USA

${ }^{8}$ Department of Surgery, University of Virginia, Charlottesville, Virginia, USA

${ }^{9}$ Russell H. Morgan Department of Radiology and Radiological Science, Johns Hopkins University School of Medicine, Baltimore, Maryland, USA

${ }^{10}$ Division of Health Sciences Informatics, Johns Hopkins University School of Medicine, Baltimore, Maryland, USA

${ }^{11}$ Armstrong Institute for Patient Safety and Quality, Johns Hopkins Medicine, Baltimore, Maryland, USA

${ }^{12}$ Center for Transgender Health, Johns Hopkins Medicine, Baltimore, Maryland, USA

Collaborators Levine, Adele Brigham and Women's Hospital, Center for Surgery and Public Health One Brigham Circle 1620 Tremont St Boston, Massachusetts, USA 021206175257300

Contributors $\mathrm{AHH}, \mathrm{BDL}$ and SP conceived the study and obtained research funding. DG, CS, ES, BDL and AHH designed and supervised the conduct of the study. LMK, RYS and AR undertook enrolment of participants, data acquisition and data management. LMK, RYS and DG performed data analysis and ensured data quality control. LMK drafted the manuscript, and all authors contributed substantially to its revision. All authors take full responsibility for the paper as a whole.

Funding This work is supported by a contract (AD-1306-03980) from the Patient-Centered Outcomes Research Institute (PCORI) entitled 'Patient-Centered Approaches to Collect Sexual Orientation/Gender Identity Information in the ED'.

Competing interests $\mathrm{BDL}$ is supported by the Institute for Excellence in Education Berkheimer Faculty Education Scholar Grant, a contract (CE-12-11-4489) from PCORI entitled 'Preventing Venous Thromboembolism: Empowering Patients and Enabling Patient-Centered Care via Health Information Technology', a contract (DI-1603-34596) from PCORI 'Preventing Venous Thromboembolism (VTE): Engaging Patients to Reduce Preventable Harm from Missed/Refused Doses of VTE Prophylaxis' and a grant from the AHRQ (1R01HS024547) entitled 'Individualized Performance Feedback on Venous Thromboembolism Prevention Practice', a grant from the NIH/ NHLBI (R21HL129028) entitled 'Analysis of the Impact of Missed Doses of Venous Thromboembolism Prophylaxis', and a grant from the Department of Defense entitled The Pathogenesis of Post-Traumatic Pulmonary Embolism: A Prospective Multicenter Investigation by the CLOTT Study Group. AHH is PI on a Health Services Grant from the Department of Defense through the Henry M Jackson Foundation entitled 'Epidemiology and Provider Induced Collaboration'. AHH is co-founder and equity holder in Patient Doctor Technologies Incorporated which owns and operates the website www.doctella.com.

Patient consent Obtained.

Ethics approval The Johns Hopkins Medicine Institutional Review Board in Baltimore, Maryland approved this study.

Provenance and peer review Not commissioned; externally peer reviewed.

\section{REFERENCES}

1 De Santis JP. HIV infection risk factors among male-to-female transgender persons: a review of the literature. J Assoc Nurses AIDS Care 2009;20:362-72.

2 Struble CB, Lindley LL, Montgomery K, et al. Overweight and obesity in lesbian and bisexual college women. J Am Coll Health 2010;59:51-6.

3 Buchmueller T, Carpenter CS. Disparities in health insurance coverage, access, and outcomes for individuals in same-sex versus different-sex relationships, 2000-2007. Am J Public Health 2010;100:489-95.
4 Dilley JA, Simmons KW, Boysun MJ, et al. Demonstrating the importance and feasibility of including sexual orientation in public health surveys: health disparities in the Pacific Northwest. Am J Public Health 2010;100:460-7.

5 Herbst JH, Jacobs ED, Finlayson TJ, et al. Estimating HIV prevalence and risk behaviors of transgender persons in the United States: a systematic review. AIDS Behav 2008;12:1-17.

6 Díaz RM, Ayala G, Bein E, et al. The impact of homophobia, poverty, and racism on the mental health of gay and bisexual Latino men: findings from 3 US cities. Am J Public Health 2001;91:927-32.

7 Shields R, Lau B, Haider AH. Emergency general surgery needs for lesbian, gay, bisexual, and transgender patients: are we prepared? JAMA Surg 2017;152:617-8.

8 U.S. Department of Health and Human Services. Healthy people. 2020. Available at https://www.healthypeople.gov/ (Accessed 4 Mar 2016).

9 U.S. Department of Health and Human Services. Edition Health Information Technology Certification Criteria, 2015 Edition Base Electronic Health Record Definition, And ONC Health IT Certification Program Modifications. 2015. Available at https://www.federalregister.gov/articles/2015/10/16/2015-25597/2015-editionhealth-information-technology-health-it-certification-criteria-2015-edition-base (Accessed 4 Mar 2016).

10 The Joint Commission. Advancing Effective Communication, Cultural Competence, and Patient - and Family-Centered Care for the Lesbian, Gay, Bisexual, and Transgender (LGBT) Community: a field guide. Oak Brook, IL. 2011. Available at http://www. jointcommission.org/lgbt/ (Accessed 4 Mar 2016).

11 Alper J, Feit MN, Sanders JQ. Collecting sexual orientation and gender identity data in electronic health records: workshop summary. Institute of Medicine. Washington, D.C.: The National Academies Press, 2013.

12 Haider AH, Schneider EB, Kodadek LM, et al. Emergency department query for patientcentered approaches to sexual orientation and gender identity: the EQUALITY Study. JAMA Intern Med 2017;177:819-28.

13 Glaser BG, Strauss AL. The discovery of grounded theory: strategies for qualitative research. New York: Aldine de Gruyter, 1967.

14 Watt D. On becoming a qualitative researcher: the value of reflexivity. The Qualitative Report 2007;12:82-101.

15 Bauer GR, Scheim Al, Deutsch MB, et al. Reported emergency department avoidance, use, and experiences of transgender persons in Ontario, Canada: results from a respondent-driven sampling survey. Ann Emerg Med 2014;63:713-20.

16 Brown JF, Fu J. Emergency department avoidance by transgender persons: another broken thread in the "safety net" of emergency medicine care. Ann Emerg Med 2014;63:721-2.

17 Maragh-Bass AC, Torain M, Adler R, et al. Risks, benefits, and importance of collecting sexual orientation and gender identity data in healthcare settings: A multi-method analysis of patient and provider perspectives. LGBT Health 2017:4:141-52

18 Callahan EJ, Sitkin N, Ton $\mathrm{H}$, et al. Introducing sexual orientation and gender identity into the electronic health record: one academic health center's experience. Acad Med 2015;90:154-60.

19 Makadon HJ. Ending LGBT invisibility in health care: the first step in ensuring equitable care. Cleve Clin J Med 2011;78:220-4.

20 Currier GW, Brown G, Walsh PG, et al. Screening for sexual orientation in psychiatric emergency departments. West J Emerg Med 2015;16:80-4.

21 Snowdon S. Health Care Equality Index 2013: promoting equitable \& inclusive care for lesbian, gay, bisexual and transgender patients and their families. Washington, D.C.: Human Rights Campaign Foundation, 2013.

22 Jalali S, Sauer LM. Improving care for lesbian, gay, bisexual, and transgender patients in the emergency department. Ann Emerg Med 2015;66:417-23.

23 Jalali S, Levy MJ, Tang N. Prehospital emergency care training practices regarding lesbian, gay, bisexual, and transgender patients in Maryland (USA). Prehosp Disaster Med 2015;30:163-6

24 Moll J, Krieger P, Moreno-Walton L, et al. The prevalence of lesbian, gay, bisexual, and transgender health education and training in emergency medicine residency programs: what do we know? Acad Emerg Med 2014;21:608-11. 